\title{
Inhibition of release of lentivirus particles with incorporated human influenza virus haemagglutinin by binding to sialic acid-containing cellular receptors
}

\author{
Valerie Bosch, ${ }^{1}$ Beatrice Kramer, ${ }^{1} \dagger$ Tanya Pfeiffer, ${ }^{1}$ Lilian Stärck ${ }^{1} \ddagger$ and David A. Steinhauer ${ }^{2}$ \\ 1 Forschungsschwerpunkt Angewandte Tumorvirologie, FO200, Deutsches Krebsforschungszentrum, Im Neuenheimer Feld 242, \\ 69120 Heidelberg, Germany \\ ${ }^{2}$ Division of Virology, National Institute for Medical Research, The Ridgeway, Mill Hill, London NW7 1AA, UK
}

\begin{abstract}
Mutants of the haemagglutinin (HA) gene of human influenza virus A/Aichi/2/68 (H3N2) encoding $\mathrm{HA}$ proteins that are proteolytically cleaved intracellularly, defective in binding to cellular receptors or defective for acylation within the cytoplasmic $C$ terminus have been generated. Here, the properties of these mutated HA molecules are described and their incorporation into the lipid membrane of released human immunodeficiency virus (HIV)-like particles is analysed. It is demonstrated that, when produced from cells coexpressing any of the binding-competent Aichi-HA molecules, release of HIV-like particles into the extracellular medium is reduced and the particles that are released fail to incorporate Aichi-HA. These blocks in release and incorporation, respectively, can both be overcome. The release of normal amounts of particles with incorporated $\mathrm{HA}$ can be achieved either by mutation of the receptor-binding site on the Aichi-HA molecule or by removal of sialic acid from surface proteins with neuraminidase. In contrast, as a result of blockage of the sialic acid-binding site by sialidated oligosaccharides on the HA itself, the HA of influenza virus $A / F P V / R o s t o c k / 34$ (H7N1) is efficiently incorporated into HIV-like particles. These results, namely that particle release can be inhibited by interactions between the incorporated glycoprotein and the cell surface and/or that interactions with other cellular components can be inhibitory to incorporation into retrovirus envelopes, probably reflect general principles that may hold for many viral and cellular glycoproteins.
\end{abstract}

\section{Introduction}

In recent years, numerous analyses have aimed to elucidate the processes that are important for the production and release into the extracellular medium of retrovirus particles that have incorporated a specific glycoprotein into their lipid membrane. These studies attempt to understand the process of glycoprotein incorporation into retroviruses in general, as well as using the principles revealed to achieve the incorporation of

Author for correspondence: Valerie Bosch.

Fax +446221 424932. e-mail v.bosch@dkfz-heidelberg.de

† Present address: MRC Laboratory for Molecular Biology, University

College London, Gower Street, London WC1E 6BT, UK.

‡ Present address: Department of Hematology, Oncology and Tumor Immunology, Humboldt-University Berlin, Charite-Campus Berlin-Buch, Lindenberger Weg 80, D-13125 Berlin, Germany. specific proteins of interest, such as those proteins necessary for retrovirus targeting. Protein incorporation is not specific to the homologous viral glycoprotein: many heterologous viral glycoproteins have been shown to be incorporated into retrovirus particles and to mediate infectivity in the appropriate host cells (pseudotyping). Additionally, and especially in the case of human immunodeficiency virus type 1 (HIV-I), numerous different cellular plasma membrane proteins have been demonstrated to be present in the retrovirus membrane (summarized by Bastiani et al., 1997). Although some cell surface glycoproteins appear to be preferentially incorporated (Arthur et al., 1992), evidence has also been reported that many cellular plasma membrane proteins can be incorporated into retrovirus envelopes without sorting (Hammarstedt et al., 2000). Thus, the amount of these proteins incorporated into the virus particle remains unclear and may simply reflect their relative concentration at the plasma membrane of the infected cell. On the other hand, viral glycoproteins appear to be 
enriched in the envelope of retrovirus particles. While, in some cases, an interaction between homologous viral glycoproteins and internal virus components may play a role in incorporation and enrichment, this is unlikely to apply to the incorporation of heterologous viral glycoproteins. Another possibility to account for this protein enrichment is that at least some viral glycoproteins passively diffuse to, and become enriched at, the site of virus budding due to their lack of inhibitory interactions with other cellular proteins. This model assumes that a viral glycoprotein, which by definition is 'foreign' to the cell and probably lacks a cellular function, may not interact intrinsically with, or may have evolved mechanisms to avoid interacting with, cellular structures that could prevent incorporation. Molecules that could potentially inhibit incorporation could be bulky proteins present on the inner side of the membrane, e.g. cytoskeletal proteins, or other cellular membrane proteins, which themselves undergo inhibitory interactions. In some cases, for example, when expressed in a foreign cell, cell surface proteins also appear to become enriched into the envelope of membrane viruses. Examples include human CD4 expressed in quail cells and efficiently incorporated into avian leukosis virus particles (Young et al., 1990), human CD4 and CXCR-4 incorporated into vesicular stomatitis virus (Schnell et al., 1997) and Cterminally truncated human epidermal growth factor receptor (EGR-R) incorporated into HIV-like particles (Henriksson et al., 1999). Another factor that may contribute to the ability of a particular glycoprotein to be incorporated into HIV particles concerns the localization of the respective glycoprotein to detergent-insoluble glycolipid-enriched membrane rafts in the plasma membrane (Nguyen \& Hildreth, 2000). In addition to many glycosylphosphatidylinositol-anchored proteins, some integral membrane proteins, e.g. influenza virus haemagglutinin (HA) and neuraminidase (NA), preferentially localize to these membrane rafts. In these latter cases, the protein features that mediate localization to these rafts are presently under investigation. For the HA, evidence has been reported that the membrane-anchor region (Scheiffele et al., 1997) as well as the three palmitoylated cysteine residues in the cytoplasmic tail of the HA may contribute to raft localization (Melkonian et al., 1999; Zhang et al., 2000).

In addition to the HA, which mediates virus entry by interacting with cellular receptors that carry oligosaccharides with terminal sialic acid residues, influenza virus encodes another surface glycoprotein, NA. NA plays a role at the end of the virus replication cycle by removing sialic acid residues from both cellular receptors and HA, thus preventing virus particles from aggregating both to each other and to the cell surface (Liu et al., 1995). Efficient incorporation of the HA of the avian influenza virus A/FPV/Rostock/34 (H7N1) (FP-HA) into the membrane of avian and murine retroviruses, which are released into the extracellular medium, has been shown previously (Dong et al., 1992; Hatziioannou et al., 1998) and, as demonstrated here, this also occurs in lentivirus (HIV) membranes. We have been interested in achieving the incorporation of, and eventually pseudotyping with, human influenza virus HA [A/Aichi/2/68 (H3N2), Aichi-HA)] into virus particles: the atomic structure (Bullough et al., 1994; Wilson et al., 1981) as well as numerous defined mutants of Aichi-HA are available. Our long-term aim is to generate pseudotyped retrovirus particles with incorporated HA proteins that are functional in membrane fusion but lack the capacity to bind sialic acid. Targeting of pseudotyped particles to specific cells or tissue types could then be specified by alternative, coincorporated surface proteins. In order to manifest fusion activity, the HA molecule must be proteolytically cleaved into the disulphide-linked subunits HAI and HA2. Human influenza virus HA molecules are not intracellularly processed, since there is only a single arginine residue at the proteolytic cleavage site between HA1 and HA2, whereas HA molecules with polybasic tracts at the HA1-HA2 junction are cleaved intracellularly in nearly all cell types. Thus, we have generated mutated Aichi-HA genes encoding potentially cleavable HA molecules, either with or without further mutations, leading to defective sialic acid receptor binding or affecting the cytoplasmic C-terminal cysteine residues implicated to influence raft localization. In this study, we describe the properties of these mutated Aichi-HA molecules, in particular, with respect to their incorporation into released HIV-like particles. Our findings indicate that, in contrast to FP-HA, HIV-like particles produced in cells coexpressing binding-competent Aichi-HA are released into the extracellular medium in reduced amounts and, further, that these released particles have failed to incorporate Aichi-HA. This situation could be overcome either by introducing mutations into Aichi-HA that abolish receptor binding or by removing sialic acid residues from cell surface proteins by treatment of cells with NA.

\section{Methods}

- Constructs. The cDNA sequence encoding the HA of influenza virus A/Aichi/2/68 (H3N2) was initially cloned into a Bluescript vector. The insertion of nucleotides encoding four additional arginines at the proteolytic cleavage site between HA1 and HA2 was carried out using standard PCR fusion techniques (Ho et al., 1989; Horton et al., 1989). The oligonucleotide used to insert the arginines was 5' 1011 GAATGTACCAGAGAAACAAACTAGGAGGA GGAGGAG AGGTCTATTCGGCGCAATAG $10543^{\prime}$. The numbers refer to the HA nucleotide positions, the letters in italics indicate the inserted nucleotides and the underlined letter indicates the position of an additional silent mutation, which destroyed an StuI restriction site and allowed mutant clones to be easily identified. The entire fragment generated by PCR was sequenced to confirm that only the desired nucleotide changes had been made. The cDNAs for the Aichi wild-type HA (Aichi-HA-Wt) and the derivative with the four inserted arginines (Aichi-HA-I4+) were then inserted into the eukaryotic expression vector pKEx (Rittner et al., 1991) to yield pKEx-Aichi-HA-Wt and pKEx-AichiHA-I4 +, respectively. A fragment (AgeI at position 1541 to a PstI site in the polylinker downstream from the HA gene) encoding the C-terminal 
cytoplasmic region of the mutated Aichi-HA with the changes $C^{538} \mathrm{M}$, $C^{545} \mathrm{~A}$ and $\mathrm{C}^{548} \mathrm{Y}$ (abbreviated MAY) (Lin et al., 1997) was exchanged for the same fragment in pKEx-Aichi-HA-I4 + to yield the derivative pKExAichi-HA-I4 + /MAY. The mutations $\mathrm{Y}^{98} \mathrm{~F}$ and $\mathrm{L}^{194} \mathrm{~A}$, described previously by Martin et al. (1998), which each alone very significantly reduce the cell receptor binding of HA, were introduced by subcloning, either alone or in combination, into pKEx-Aichi-HA-I4 + to yield pKExAichi-HA-I4 + /Y98F, pKEx-Aichi-HA-I4 + /L194A and pKEx-AichiHA-I4 + /DBM (DBM for double binding-defective mutant), respectively. The cDNAs for the mutated HA molecules were additionally cloned into the vaccinia virus transfer plasmid PRB2I and recombinant vaccinia viruses expressing the mutated proteins were generated, essentially as described previously (Blasco \& Moss, 1995). The cDNA encoding the FP-HA protein was also cloned into pKEx to yield pKEx-FPHA. An expression plasmid for bovine furin, pSG5new/bFur (Vey et al., 1994), referred to here as pEx-furin, and a plasmid containing the cDNA for the NA gene from influenza virus A/WSN/33 (H1N1) were kind gifts from H.-D. Klenk and W. Garten, University of Marburg, Germany. The cDNA for the NA gene was cloned into pKEx to yield pKEx-NA. pKExHIV $\Delta$ Env3, which encodes all of the HIV gene products except Env and Nef and leads to the release of non-infectious HIV-like particles, has been described previously (Henriksson \& Bosch, 1998).

Analyses of HA expression and incorporation into HIV-like particles. Human 293 T cells (Pear et al., 1993), maintained in Dulbecco's modified Eagle's medium with $10 \%$ foetal calf serum, were transfected by standard calcium-phosphate transfection procedures. Transfected cells were metabolically labelled with $75 \mu \mathrm{Ci} / \mathrm{ml}\left[{ }^{35} \mathrm{~S}\right]$ methionine $/\left[{ }^{35} \mathrm{~S}\right]$ cysteine (Amersham) for $12 \mathrm{~h}$ from 36 to $48 \mathrm{~h}$ post-transfection (p.t.) in methionine/cysteine-depleted serum-free medium. When required, the labelling media were supplemented with $0.05 \mathrm{U} / \mathrm{ml} \mathrm{NA}$ from Vibrio cholerae (Dade Behring). Labelled cells were lysed with 1\% Triton-X-100 in PBS supplemented with protease inhibitor cocktail (Boehringer). After clarification, cell lysates were adjusted to $1 \%$ Triton-X-100, $0.5 \%$ deoxycholate and $0.1 \%$ SDS in PBS supplemented with protease inhibitor cocktail (RIPA buffer). Metabolically labelled HIV-like particles, released into the culture supernatants of cells transfected with pKEx-HIV $\Delta$ Env3, were pelleted by centrifuging the filtered media $(0 \cdot 45 \mu \mathrm{m}$ pore-size filter) through a cushion of $32 \%$ sucrose, as described previously (Henriksson \& Bosch, 1998; Henriksson et al., 1999). Pelleted materials were lysed in a small volume of $1 \%$ Triton-X-100 in PBS and either analysed directly by PAGE or adjusted to RIPA buffer detergent concentrations for immunoprecipitation. Immunoprecipitation was carried out, essentially as described previously (Pfeiffer et al., 1997), by employing polyclonal antiserum against bromelain-digested Aichi-HA (anti-Aichi-HA), rabbit anti-FPV serum reactive against FP-HA (anti-FP-HA) (a kind gift from H.-D. Klenk and W. Garten, University of Marburg, Germany) or rabbit antiserum against the HIV capsid (CA) protein p24 (anti-HIV p24). The amount of HIV-like particles released into the culture supernatants was quantified by ELISA detecting HIV-I CA (Innogenetics).

Antibody recognition, receptor-binding and membrane fusion activities. The cell surface expression levels and conformations of the wild-type and mutant HA molecules were analysed by ELISA using a panel of monoclonal antibodies (MAbs) to different structural regions of the HA in HeLa cells infected with the respective recombinant vaccinia viruses, as described previously (Martin et al., 1998; Steinhauer et al., 1991). The assay for HA receptor-binding activity has also been described previously by Martin et al. (1998). Briefly, human erythrocytes loaded with horseradish peroxidase (HRP) were added to recombinant vaccinia virus-infected HeLa cells expressing the respective HA proteins. After washing, the concentration of HRP remaining was determined and the results corrected for HA expression, as established by ELISA using polyclonal rabbit anti-Aichi serum. The assay for membrane-fusion activity was also performed essentially as described previously (Martin et al., 1998). BHK-21 cells were infected with recombinant vaccinia viruses expressing the respective HA proteins and, at $15 \mathrm{~h}$ post-infection (p.i.), cells were treated with a low $\mathrm{pH}(5 \cdot 0)$ buffer for $1 \mathrm{~min}$. After neutralization, incubation was continued for $30 \mathrm{~min}$ and the cells were fixed, stained and photographed.

\section{Results}

\section{Expression and proteolytic processing of wild-type and mutated HA proteins}

Fig. 1 illustrates the relevant features of the wild-type and mutant HA proteins used in this study. In common with all other human influenza virus HA proteins, the HA from the Aichi strain contains only a single arginine residue at the cleavage site between HA1 and HA2 and, thus, is not proteolytically processed intracellularly in the trans-Golgi network. In the case of another HA of the same subtype, influenza virus A/Port Chalmers/1/73, it has been demonstrated previously that a mutated HA protein with four arginine residues inserted at the cleavage site was efficiently processed intracellularly and was fusogenic after low $\mathrm{pH}$ treatment (Ohuchi et al., 1991). Thus, in order to more easily monitor intracellular transport and to generate a functional Aichi-HA protein in the absence of trypsin treatment, we generated pKEx-Aichi-HA-I4+, which has four arginine residues inserted at the cleavage site, and compared its expression to that of the wild-type construct pKEx-Aichi-HAWt and, additionally, pKEx-FP-HA, which encodes intracellularly cleavable FP-HA. Transfected 293T cells were metabolically labelled for approximately $12 \mathrm{~h}$ at $36-48 \mathrm{~h}$ p.t. and the respective HA molecules were immunoprecipitated from cell lysates and analysed by gel electrophoresis (Fig. 2). As expected, the FP-HA protein was intracellularly cleaved into HA1 (5I kDa) and HA2 (28 kDa) and only a minor band representing the $\mathrm{HA}$ precursor $\mathrm{HAO}(80 \mathrm{kDa})$ could be detected (Fig. $2 \mathrm{~A}$, lane 1). On the other hand, the precursor HAO $(82 \mathrm{kDa})$ from Aichi-HA-Wt was not proteolytically processed. In the case of the mutant Aichi-HA-I4+, HAI $(56 \mathrm{kDa})$ and HA2 $(26 \mathrm{kDa})$ were clearly detected, indicating that the insertion of the four arginine residues had in fact generated a cleavage site for the cellular protease expressed in the 293 T cells. However, in contrast to FP-HA, the relative amount of unprocessed HA0, in comparison to HA1 plus HA2, was much higher, indicating that intracellular cleavage was suboptimal (Fig. 2 A). Since efficient proteolytic processing is a prerequisite for HA function, we attempted to increase this by coexpressing an additional processing protease in cells expressing Aichi-HA-I4 + . For this purpose, pEx-furin, which encodes bovine furin (shown to be able to process influenza virus HA) (Vey et al., 1994), was employed. As shown in Fig. 2(B), Aichi-HA-I4 + was clearly proteolytically processed more efficiently in the presence of bovine furin, while AichiHA-Wt remained uninfluenced. Quantification of the radio- 

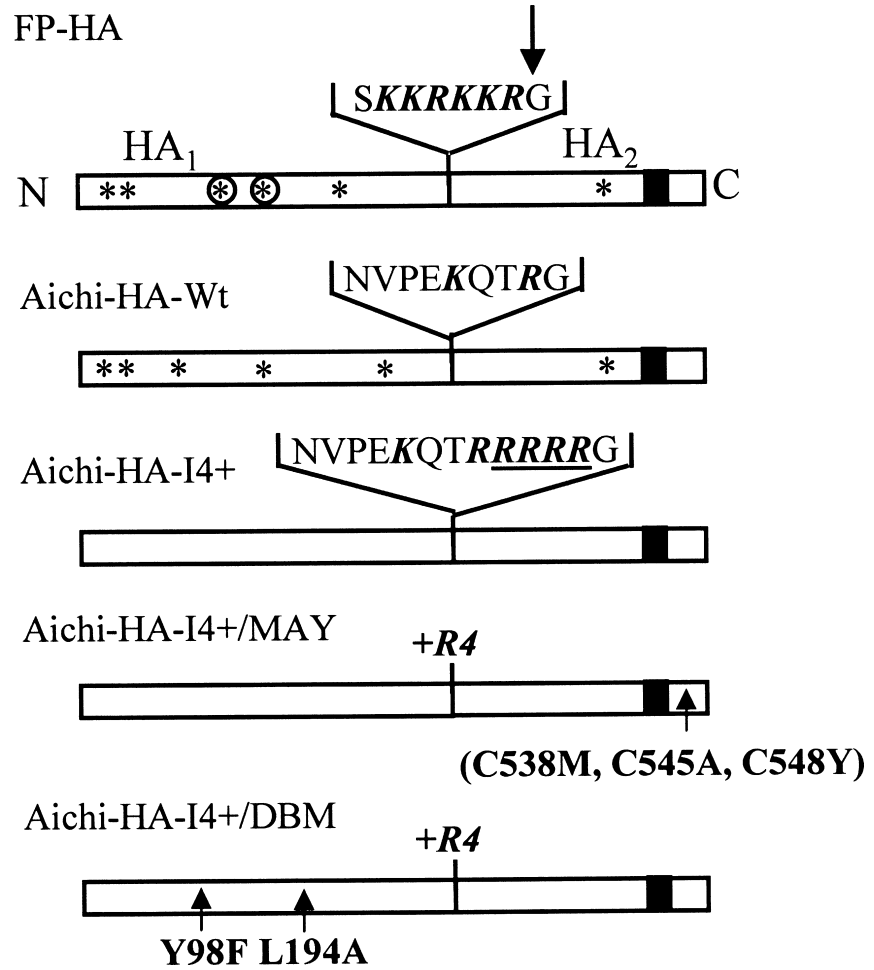

Fig. 1. Relevant features of the HA constructs employed. The HA proteins are shown as open bars, with a vertical line depicting the border between HA1 and HA2. Filled bars depict the membrane-spanning domain within HA2. The amino acid sequences at the cleavage site of the wild-type HA proteins are given, with the exact cleavage site indicated with an arrow. The approximate positions of $N$-linked oligosaccharides are shown by asterisks. The two oligosaccharides that affect receptor-binding of FP-HA are circled. The amino acid sequence at the cleavage site of the cleavable mutant Aichi-HA-14 + is shown with the inserted amino acids underlined. The positions of mutations within the cytoplasmic C-terminal domain of HA2 in Aichi-HA-I4 + /MAY are shown. The positions of the mutations that inhibit receptor binding are also shown for Aichi-HA-I4 + /DBM. In both Aichi-HA-14 + /MAY and Aichi-HA-14 + /DBM, the inserted arginines are indicated as + R4.

activity in respective bands revealed that the percentage of total HA in HAO in comparison to HAI plus HA2 was $44 \%$ in the absence, and $10 \%$ in the presence, of bovine furin. That is, in this latter case, the efficiency of proteolytic processing was similar to that observed when FP-HA was expressed (Fig. 2 A, lane 1).

\section{Incorporation of binding-competent HA proteins into HIV-like particles released into the extracellular medium}

293T cells were transfected with expression vectors for the binding-competent HA proteins FP-HA, Aichi-HA-I4+ and Aichi-HA-I4 + /MAY (the latter HA protein contains mutations at three cysteine residues in the cytoplasmic $\mathrm{C}$ terminus) in the presence or absence of HIV-like particle expression from pKEx-HIV $\Delta$ Env3. Expression of the respective HA molecules and of HIV-CA in transfected cells was confirmed both by immunofluorescence and by immuno-
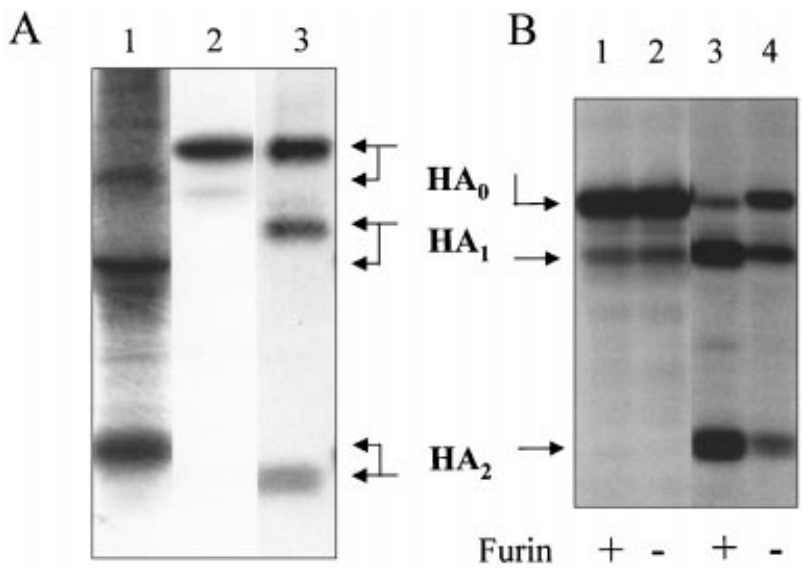

Fig. 2. Proteolytic processing of the HA protein. (A) PAGE of HA proteins immunoprecipitated from metabolically labelled 293T cells transfected with pKEx-FP-HA (lane 1), pKEx-Aichi-HA-Wt (lane 2) or pKEx-Aichi-HA$14+$ (lane 3). The positions of HAO, HA1 and HA2 are shown on the right. (B) PAGE of HA proteins immunoprecipitated from metabolically labelled 293T cells transfected with pKEx-Aichi-HA-Wt (lanes 1 and 2) or pKEx-Aichi-HA-14 + (lanes 3 and 4), either with (+, lanes 1 and 3 ) or without (-, lanes 2 and 4 ) cotransfection with pEx-furin. In the case of the cell lysates expressing Aichi-HA-Wt (lanes 1 and 2), the identity of the weaker bands migrating slightly slower than HA1 is not known. The positions of HAO, HA1 and HA2 are shown on the left.
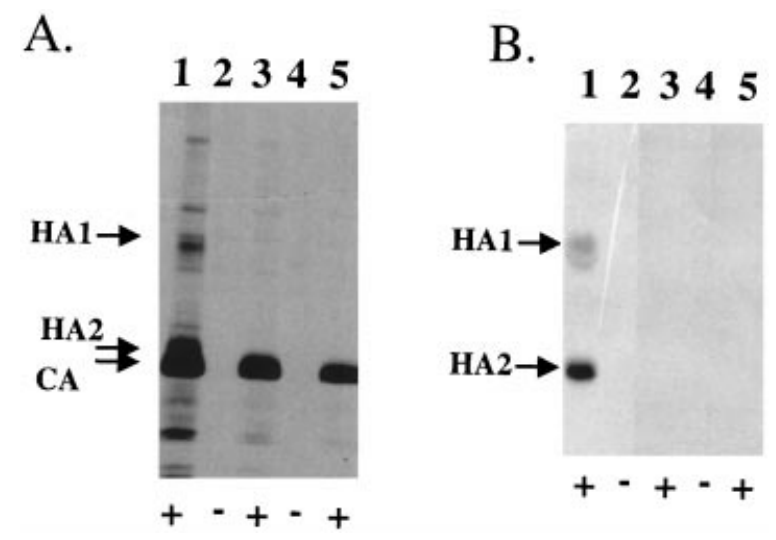

Fig. 3. Incorporation of binding-competent HA into HIV-like particles. Pelleted materials were prepared from metabolically labelled 293T cells transfected with pKEx-FP-HA (lane 1), pKEx-Aichi-HA-14 + (lanes 2 and 3 ) or pKEx-Aichi-HA-I4 + /MAY (lanes 4 and 5), either with (+) or without $(-)$ pKEx-HIV $\Delta$ Env3. (A) Direct PAGE (no prior immunoprecipitation) of centrifuged pellets from the culture supernatants of transfected cells. (B) PAGE of HA proteins immunoprecipitated from the lysates of the centrifuged pellets analysed directly in $(A)$. The positions of the relevant HA and HIV proteins are shown.

precipitation (data not shown). The pelleted materials, generated by centrifuging the cultured supernatants through a cushion of sucrose, were analysed first by direct electrophoresis without prior immunoprecipitation. As shown in Fig. 3(A), HIV structural proteins (HIV-CA is the major component) were detected only in those samples in which pKEx-HIV $\Delta$ Env3 had been expressed. In the case of particles released from cells coexpressing FP-HA, radioactive bands representing FP-HA1 and $-\mathrm{HA} 2$ were detected in the absence of specific anti-HA 
Table 1. Properties of the wild-type and mutant Aichi-HA molecules

\section{(A) MAb reactivity}

The reactivity of MAbs to intact cells expressing the mutant HA proteins by ELISA is shown. MAbs were designated previously by Daniels et al. (1983).

\begin{tabular}{|c|c|c|c|c|c|}
\hline Construct & $\mathrm{HC} 3$ & $\mathrm{HC} 31$ & $\mathrm{HC} 68$ & $\mathrm{HC} 100$ & HC263 \\
\hline Aichi-HA-Wt & $1 \cdot 188$ & 0.686 & 0.551 & $0 \cdot 487$ & $0 \cdot 386$ \\
\hline Aichi-HA-I4 + & $1 \cdot 122$ & 0.646 & 0.599 & $0 \cdot 474$ & $0 \cdot 369$ \\
\hline Aichi-HA-I4 + /Y98F & $1 \cdot 453$ & $1 \cdot 132$ & $1 \cdot 335$ & 0.701 & 0.638 \\
\hline Aichi-HA-I4 + /L194A & $1 \cdot 087$ & $0 \cdot 792$ & 0.577 & $0 \cdot 427$ & $0 \cdot 356$ \\
\hline Aichi-HA-I4 + /DBM & 0.964 & 0.659 & $0 \cdot 446$ & $0 \cdot 400$ & $0 \cdot 266$ \\
\hline
\end{tabular}

(B) Human erythrocyte-binding assay

\begin{tabular}{|c|c|}
\hline Construct & $\begin{array}{c}\text { Human erythrocyte } \\
\text { binding (\% of wild-type) }\end{array}$ \\
\hline Aichi-HA-Wt & 100 \\
\hline Aichi-HA-I4 + & $95 \cdot 3$ \\
\hline Aichi-HA-I4 + /Y98F & 0.8 \\
\hline Aichi-HA-I4 + /L194A & $0 \cdot 4$ \\
\hline Aichi-HA-I4 + /DBM & $0 \cdot 0$ \\
\hline
\end{tabular}

immunoprecipitation. Fig. 3(B) shows the radioactive molecules, immunoprecipitated with the respective anti-HA sera, from lysates of the pelleted virus particles shown in Fig. 3 (A). FP-HA1 and-HA2 components were clearly detectable. These results point to genuine incorporation of FP-HA into HIV-like particles, which is in line with previous reports that demonstrate the incorporation of FP-HA into avian and murine retroviruses (Dong et al., 1992; Hatziioannou et al., 1998). In contrast, immunoprecipitated, binding-competent Aichi-HA$\mathrm{I} 4+$ or Aichi-HA-I4 + /MAY could not be detected, even after a very long exposure of the gel to film. These results indicate that these components had failed to be incorporated in detectable amounts into the respective pelleted HIV-like particles. As already shown in Fig. 3 (A) (compare the intensities of $\mathrm{CA}$ in lanes 1, 3 and 5), an additional effect of the coexpression of the binding-competent Aichi-HA-I4 + and Aichi-HA-I4 + /MAY was a reduction in the amount of HIVlike particles released into the extracellular medium. Quantification of particle release by ELISA for HIV-CA revealed that, although somewhat variable, the amount of virus particles released from cells expressing Aichi-HA-I4 + or Aichi-HAI4 + /MAY was in the range of $20 \%$ of that released when pKEx-HIV $\Delta$ Env3 was expressed either alone or with FP-HA (this did not differ significantly). This reduction was also observed, and, in fact, was more pronounced, on coexpression of non-cleavable Aichi-HA-Wt (Table 2).

\section{Further properties of cleavable Aichi-HA-I4+ and of binding-incompetent Aichi-HA-I4+/DBM}

Based on the crystal structure of Aichi-HA-receptor analogue complexes, Aichi-HA mutants with single amino acid changes predicted to inhibit sialic acid binding have been generated and analysed. Several of these mutations severely impaired binding. However, the results of a sensitive infectivity assay demonstrated that this impairment was not complete (Martin et al., 1998). With the aim of completely abolishing receptor-binding, two of these mutations, $\mathrm{Y}^{98} \mathrm{~F}$ and $\mathrm{L}^{194} \mathrm{~A}$, each of which severely impairs binding, were introduced together into the cDNA sequence encoding Aichi-HA-I4+. The mutated gene product is referred to as Aichi-HA-I4 + /DBM. In an initial assay, we determined if the different HA molecules were expressed at the cell surface and if they were reactive with a panel of five MAbs, each of which recognizes a different conformational determinant on the Aichi-HA protein. As shown in Table $I(A)$, binding of all of the MAbs to intact recombinant vaccinia virus-infected cells expressing Aichi-HAWt and Aichi-HA-I4 + was virtually identical, demonstrating that the insertion of the four arginine residues at the cleavage site had no detectable effect on the conformation of the HA. This was also the case for Aichi-HA-I4+/L194A. On the other hand, binding of all of the MAbs to cells expressing Aichi-HA-I4 + /Y98F and Aichi-HA-I4 + /DBM was slightly 
A.
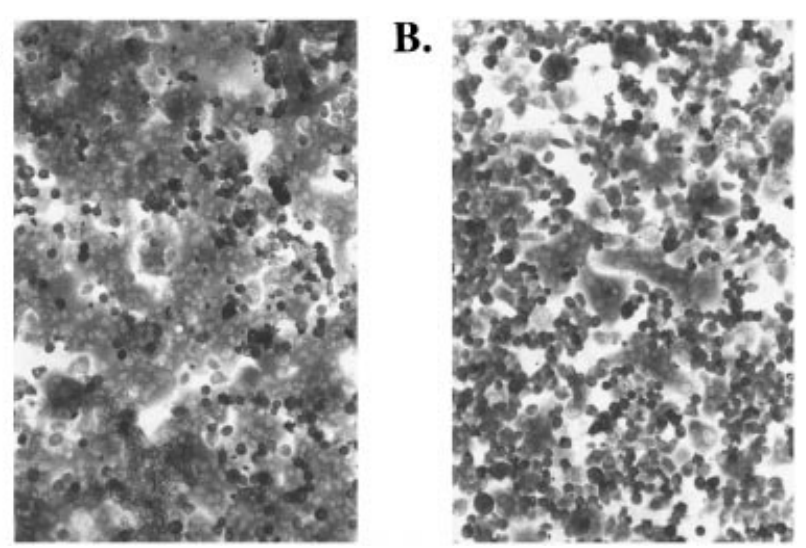

Fig. 4. Polykaryon formation after low pH treatment. BHK-21 cells infected with recombinant vaccinia viruses expressing Aichi-HA-14 + (A) or Aichi$\mathrm{HA}-\mathrm{I} 4+/ \mathrm{DBM}$ (B) were treated with low $\mathrm{pH}$ buffer and fixed and stained after a further $30 \mathrm{~min}$.

higher and slightly lower, respectively, than to Aichi-HA-Wt (Table IA). However, since all of the MAbs reacted with both constructs, the differences in binding probably reflect differences in cell surface expression rather than effects of mutations on the antigenic structure of any specific region of the respective molecules.

The receptor-binding activities of the different Aichi-HA molecules were determined in an erythrocyte-binding assay. As shown in Table $1(B)$, cells expressing Aichi-HA-I4 + bind erythrocytes equally well as those expressing Aichi-HA-Wt. As has been demonstrated previously (Martin et al., 1998), the mutations $\mathrm{Y}^{98} \mathrm{~F}$ and $\mathrm{L}^{194} \mathrm{~A}$ (here in the context of Aichi-HA$\mathrm{I} 4+$ ) virtually abolish erythrocyte binding and marginal values, only slightly higher than the background values, were measured. In the case of Aichi-HA-I4 + /DBM, no erythrocyte binding could be detected.

Since it is known that, in the absence of receptor-binding, close proximity of the target membrane is sufficient to allow low $\mathrm{pH}$-induced membrane fusion, we tested the abilities of the different HA molecules to mediate polykaryon formation after low $\mathrm{pH}$ treatment. All of the cleavable HA molecules, but not vaccinia virus alone (Steinhauer et al., 1991) (data not shown), whether competent at binding or not, did, indeed, induce polykaryon formation in BHK-2I cells. This is shown in Fig. 4 for Aichi-HA-I4 + and Aichi-HA-I4 + /DBM. Although clear syncytia can be observed in the case of the binding-defective Aichi-HA-I4+/DBM, these syncytia are less pronounced in comparison to those induced by the binding-competent Aichi-HA-I4 +. This may be a result of the lack of receptor binding and/or may be due to the slightly poorer cell surface expression observed by binding of the MAbs (Table 1A).

\section{Incorporation of binding-defective Aichi-HA into HIV-like particles}

293T cells were transfected with pKEx-Aichi-HA-I4 + / DBM or pKEx-FP-HA in the presence or absence of pKEx-
HIV $\Delta$ Env3. In a further culture, pEx-furin was cotransfected with pKEx-Aichi-HA-I4+/DBM and pKEx-HIV $\Delta$ Env3. As shown in Fig. 5(A), cellular expression of Aichi-HA-I4+ / DBM was approximately equal, independent of coexpression of HIV-like particles and bovine furin. In the presence of bovine furin, proteolytic processing of HA into HA1 and HA2 in cell lysates was again increased (Fig. $5 \mathrm{~A}$, compare lanes 2 and 3). HIV-CA was immunoprecipitated in approximately equal amounts only from samples in which pKEx-HIV $\Delta$ Env3 had been coexpressed (Fig. 5 A, lower panel). Fig. 5 (B) shows direct PAGE (no prior immunoprecipitation) of enriched HIVlike particles released from the respective cultures. HIV proteins could only be observed when pKEx-HIV $\Delta$ Env3 had been expressed. Quantification of HIV-CA by ELISA demonstrated that, in contrast to the coexpression of the binding-competent Aichi-HA molecules, coexpression of Aichi-I4+/DBM did not result in a reduction in the release of HIV-like particles. In addition to the protein bands present in all of the pelleted virus preparations, radioactive bands at the positions of Aichi-HAO and -HAI (on expression of Aichi-HA-I4 + /DBM without bovine furin), Aichi-HAI (on expression of Aichi-HA-I4+ / DBM with bovine furin) and FP-HA1 plus -HA2 (on expression of FP-HA) were observed. This indicated that these HA molecules had been incorporated. The possible presence of Aichi-HA2 $(26 \mathrm{kDa})$ could not be analysed as it was not separated from HIV-CA (24 kDa). Immunoprecipitation of HA molecules from enriched HIV-like particles is shown in Fig. 5 (C). It can now be seen more clearly that, in the absence of bovine furin, Aichi-HA-I4 + /DBM HAO, HA1 and HA2 had been incorporated, whereas HA1 and HA2 were the predominant incorporated molecules in the presence of bovine furin. No HA-specific bands were observed in the absence of HIV-like particle expression. This experiment clearly demonstrated that, in contrast to the binding-competent Aichi-HA proteins analysed in Fig. 3, Aichi-HA-I4 + /DBM could be efficiently incorporated into HIV-like particles. Since in the presence of bovine furin, predominantly cleaved HAI and HA2 exit the cell in released HIV-like particles, this at least partially explains why the change in the intracellular ratio of HAO to HA1 plus HA2 (Fig. $5 \mathrm{~A}$ ), in the presence of furin, was not as dramatic as that observed in Fig. 2. In this latter case, expression of intracellular Aichi-HA-I4 + had been analysed in the absence of HIV-like particles. It is important to note that in the absence, but not in the presence, of coexpressed bovine furin, mixtures of HA-trimers containing both processed and unprocessed HA molecules may exist in the retrovirus envelope and are probably inhibited in HA function.

\section{Effect of NA on incorporation of binding-competent HA proteins into HIV-like particles}

By analogy to the situation with NA-defective influenza virus (Liu et al., 1995), we considered the possibility that interactions with sialidated surface proteins were resulting in the reduced release of HIV-like particles with incorporated 


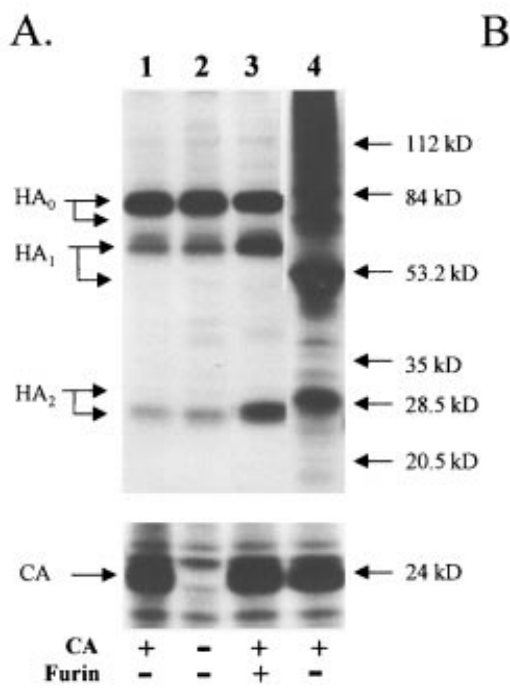

B.

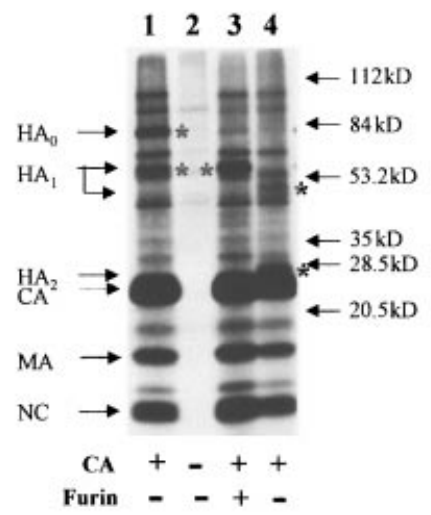

C. $\quad \begin{array}{llll}1 & 2 & 3 & 4\end{array}$

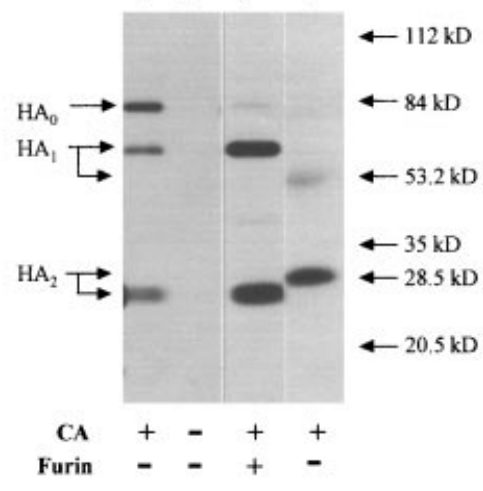

Fig. 5. Incorporation of binding-incompetent Aichi-HA-I4 + /DBM into HIV-like particles. Metabolically labelled 293T cells cotransfected with pKEx-Aichi-HA-I4 + /DBM (lanes 1-3) or pKEx-FP-HA (lane 4), either with (+) or without (-) pKExHIVAEnv3 (lanes 1, 3 and 4; designated CA) and pEx-furin (lane 3). (A) PAGE of HA (upper panel) and HIV-CA (lower panel) immunoprecipitated from lysates of transfected cells. (B) Direct PAGE (no prior immunoprecipitation) of centrifuged pellets from the culture supernatants of transfected cells. Asterisks highlight the positions of incorporated HA proteins. (C) PAGE of HA proteins immunoprecipitated from the lysates of the centrifuged pellets analysed directly in (B). The positions of the relevant HA and HIV proteins are shown on the left. Molecular mass markers are shown on the right.

Table 2. Effect of NA on the release of HIV-like particles from cells coexpressing Aichi-HA

The amount of HIV-like particles released into the medium of transfected cells treated either with or without soluble NA between 36 and 48 h p.t. was quantified by ELISA for HIV-CA. The amount of virus particles released from cells coexpressing Aichi-HA-I4 + /DBM was taken as $100 \%$.

\begin{tabular}{|lcc|}
\hline & \multicolumn{2}{c|}{ Virus release (\%) } \\
\cline { 2 - 3 } Construct & Without NA & With NA \\
\hline \multirow{2}{*}{ Aichi-HA-I4 + /DBM } & 100 & 100 \\
Aichi-HA-I4 + & 28 & 100 \\
Aichi-HA-I4 + /MAY & 28 & 110 \\
Aichi-HA-Wt & 1 & 72 \\
& & \\
\hline
\end{tabular}

binding-competent Aichi-HA and, additionally, were responsible for the fact that those particles that were released did not reveal detectable incorporation of the binding-competent Aichi-HA molecules. To examine this, we added bacterial NA, which removes sialic acid from glycoproteins and glycolipids, during metabolic labelling. The first observation that we made was that the addition of NA resulted in an increase in the amount of HIV-like particles released from cells coexpressing binding-competent Aichi-HA. As illustrated in Table 2, in the presence of NA, the amounts of HIV-like particles released were now approximately equivalent to those released from

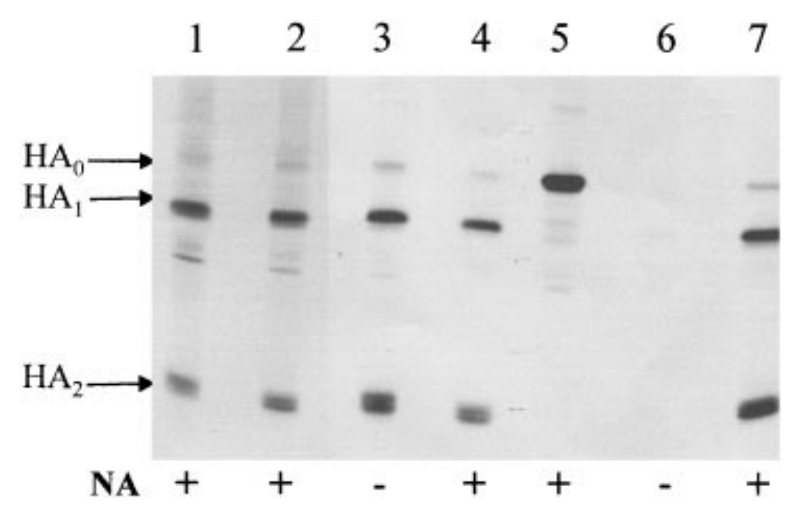

Fig. 6. Incorporation of Aichi-HA proteins in the presence of NA. PAGE of HA proteins immunoprecipitated from centrifuged pellets from the culture supernatants of 293T cells cotransfected with pKEx-Aichi-HA-14 + plus pKEx-NA (lane 1), pKEx-Aichi-HA-14 + (lane 2), pKEx-Aichi-HA-I4 + /DBM (lanes 3 and 4), pKEx-Aichi-HA-Wt (lane 5) or pKEx-Aichi-HA-I4 + /MAY (lanes 6 and 7), in all cases with pKEx-HIV $\Delta$ Env3 and pEx-furin. NA from Vibrio cholerae was added to the culture supernatants of the samples analysed in lanes 2, 4, 5 and 7. Samples with NA (+), either expressed from pKEx-NA or soluble, and samples without NA (-) are shown. The positions of HAO, HA1 and HA2 are shown on the left.

cells expressing pKEx-HIV $\Delta$ Env3 alone or coexpressing AichiHA-I4 + /DBM. This was also the case on coexpression of Aichi-HA-Wt, which, in the absence of NA, had virtually completely abolished HIV-like particle release. Fig. 6 shows the Aichi-HA components immunoprecipitated from lysates of pelleted particles produced in the presence of soluble NA. The binding-competent Aichi-HA-Wt, Aichi-HA-I4 + and AichiHA-I4 + /MAY proteins were incorporated into enriched HIV- 
particles in amounts similar to that of the binding-incompetent Aichi-HA-I4 + /DBM. Incorporation of Aichi-HA-I4 + /DBM was equal in the presence or absence of soluble NA. Aichi-HA was also observed if, instead of adding soluble NA to the culture medium, the NA gene from influenza virus WSN was coexpressed from pKEx-NA. This is illustrated for Aichi-HAI + in Fig. 6. It can be seen again (Fig. 6, lane 6) that, in the absence of NA, Aichi-HA-I4 + /MAY is not incorporated into released virions. These results show that destruction of sialic acid-containing cellular-binding partners allows the release of HIV-like particles that have efficiently incorporated bindingcompetent Aichi-HA molecules.

\section{Discussion}

Due to the availability of detailed structural information, the Aichi-HA glycoprotein, and defined variants thereof, represent interesting candidates for pseudotyping retrovirus vectors. Two absolute requirements for functional pseudotyping are proteolytic processing and incorporation of the HA into the retrovirus envelope, issues which have been addressed in this study. By analogy to a similar mutant generated in the HA gene of the related human influenza virus A/Port Chalmers/1/73 (Ohuchi et al., 1991), we generated the potentially cleavable variant Aichi-HA-I4 +, which has four arginine residues inserted at the cleavage site. However, proteolytic processing of this variant in $293 \mathrm{~T}$ cells, although clearly detectable, was suboptimal (Fig. 2A); proteolytic processing could not be increased by the introduction of another arginine residue at the cleavage site (designated AichiHA-I5 + ; data not shown). However, on coexpression of bovine furin, cleavage of Aichi-HA-I4 + was substantially improved and reached levels comparable to those observed for avian FP-HA. Thus, the suboptimal cleavage observed on expression of Aichi-HA-I4+ alone was due neither to inadequate intracellular transport nor to inaccessibility of the cleavage site. In fact, additional analyses with a panel of MAbs also confirmed unimpeded cell surface expression and correct conformation (Table IA). Thus, suboptimal cleavage is more likely to be the result of poor recognition of the Aichi-HAI + cleavage site by the processing enzymes endogenously expressed in $293 \mathrm{~T}$ cells. The proteolytic processing properties of the variant Aichi-HA-I4+/MAY, in which the three palmitoylated cysteine residues in the cytoplasmic tail of the HA protein had been mutated, were indistinguishable from those of Aichi-HA-I4 + . The mutation of these residues in the context of Aichi-HA-Wt does not affect the ability of the Aichi-HA to form heterokaryons and, in reverse genetic experiments, the mutated HA protein is incorporated into infectious viruses (Lin et al., 1997; Zhang et al., 2000).

The binding-defective mutant Aichi-HA-I4+/DBM generated in this study carries two amino acid changes, $Y^{98} \mathrm{~F}$ and $\mathrm{L}^{194} \mathrm{~A}$, each of which individually significantly inhibits binding to cellular receptors (Martin et al., 1998). These amino acid changes have not led to protein misfolding (Table 1) and proteolytic processing is the same as that observed for Aichi$\mathrm{HA}-\mathrm{I} 4+$. Further proof of protein integrity is the fact that, despite the complete lack of detectable receptor binding (Table IB), Aichi-HA-I4 + /DBM was functional in inducing membrane fusion after low $\mathrm{pH}$ treatment (Fig. 4). This demonstrated, as has also been shown by others (Ellens et al., 1990; Schoen et al., 1996; Wharton et al., 1986), that proximity to the target membrane (in this case of a neighbouring BHK-2I cell) is sufficient to allow low $\mathrm{pH}$-induced membrane fusion in the absence of sialic acid receptor binding.

Coexpression of any of the binding-competent Aichi-HA molecules in cells transfected with the HIV particle expression vector pKEx-HIV $\Delta$ Env3 led to a reduction in the quantity of HIV-like particles released (Table 2). In the cases of Aichi-HAI4 + and Aichi-HA-I4 + /MAY, however, particle release was clearly still occurring (it was about $20 \%$ compared to that observed in the absence of HA), but the particles that were released did not reveal any detectable incorporated HA molecules. In contrast, the extent of HIV-like particle release was normal on coexpression of the binding-defective mutant Aichi-HA-I4 + /DBM and returned to normal when cells expressing any of the binding-competent HA molecules were treated with soluble NA from $V$. cholerae. In these latter cases, the released particles also revealed efficient incorporation of the respective Aichi-HA proteins. On the one hand, these results strongly support the concept that interactions between binding-competent Aichi-HA and other sialic acid-containing components are the reason for the reduction in particle release. On the other hand, they also explain the lack of incorporation of Aichi-HA molecules in the remaining released particles. Liu et al. (1995) have reported similar findings with NA-deficient influenza virus. They could demonstrate that, in the lack of NA, influenza virus particles remain associated with the infected cell, to some extent, as aggregates at the cell surface and, to some extent, after re-entry, as aggregates within intracellular vesicles. The association of these influenza viruses with the cell is a consequence of an association of the HA protein in virions with other sialic acid molecules, these being either other HA molecules, with resulting virus aggregation, or sialic acidcontaining cellular receptors. Virus release and infectivity could be restored by the addition of soluble NA to the extracellular medium. It is very likely that the same phenomenon is responsible for the reduction in HIV-like particle release from cells expressing binding-competent Aichi-HA. In other words, virions that are potentially releasable, which presumably contain incorporated binding-competent Aichi$\mathrm{HA}$, remain associated with the cell and are not found in the extracellular medium unless NA, either coexpressed in the cell or in a soluble form in the medium, is present. The fraction of particles in the extracellular media of cells coexpressing Aichi$\mathrm{HA} / \mathrm{I} 4+$ or Aichi-HA/I4 + /MAY are presumably released since they have failed to incorporate detectable amounts of the 
respective binding-competent Aichi-HA. We can only speculate in general terms as to the reason for this lack of incorporation. It is conceivable that the released particles have budded from regions of the membrane that per se lack bindingcompetent Aichi-HA. Alternatively, it may be that interactions with certain sialic acid-containing cellular molecules may have resulted in a segregation of the binding-competent Aichi-HA molecules away from the budding site of that fraction of particles that are still released.

As shown in Table 2, non-cleavable Aichi-HA-Wt results in a more pronounced inhibition of HIV-like particle release than the cleavable Aichi-HA-I4 + and Aichi-HA-I4 + /MAY. We do not have an explanation for this. Although one could speculate that more avid binding to sialic acid-containing molecules could account for this difference, we did not observe any differences in receptor binding, as measured in an erythrocyte-binding assay (Table I A). The three palmitoylated cysteine residues in the cytoplasmic tail of the HA protein, which have been mutated in Aichi-HA-I4 + /MAY, have been reported to reduce the association of Aichi-HA with glycolipidenriched membrane rafts (Melkonian et al., 1999; Zhang et al., 2000). However, in the presence of NA, the extent of incorporation appeared to be comparable to that observed with Aichi-HA-I4 + , indicating that the altered localization has not affected incorporation.

The fact that FP-HA was incorporated into HIV-like particles is consistent with the previously reported efficient incorporation of FP-HA into the membrane of released avian and murine retroviruses (Dong et al., 1992; Hatziioannou et al., 1998). However, in light of the results with the bindingcompetent Aichi-HA molecules shown in this study, an open question remains as to why this is the case. Ohuchi et al. (1995, 1997) have demonstrated that, in the absence of NA, two FPHA-sialidated oligosaccharides attached to the asparagine residues at positions 123 and 149 , which are in the vicinity of the receptor site, inhibit binding of FP-HA to sialic acidcontaining receptors and, thus, its haemagglutinating activity. In other words, although FP-HA is a wild-type molecule, receptor-binding function is inhibited in the absence of NA. Thus, it is logical to postulate that the blockage of the receptorbinding site is the reason why FP-HA can be incorporated into retrovirus particles in the absence of NA. The relative positions of the attached $\mathrm{N}$-linked oligosaccharides in Aichi-HA are different (Fig. 1). The equivalent of that attached to FP-HA $\mathrm{N}^{123}$ is missing and the equivalent of that attached to $\mathrm{N}^{149}$ is shifted by a few amino acids. These Aichi-HA oligosaccharides apparently do not block sialic acid-containing receptor binding of the Aichi-HA protein.

The principles concerning virus particle release and glycoprotein incorporation, which have been learnt from these analyses with influenza virus HA, are, in fact, more generally applicable. An example would be the trapping of HIV on the surface of dendritic cells by DC-SIGN, a process of pronounced functional importance in vivo (Geijtenbeek et al., 2000).
Furthermore, in a scenario similar to that described in this study, incorporation of HIV-Env into released particles was blocked by interactions with the cellular receptor CD4, a situation that, in this case, was reversible by coexpression of HIV-Nef and -Vpu (Lama et al., 1999). These considerations should be kept in mind when attempts aimed at the incorporation of a particular glycoprotein into, for example, retrovirus vector particles are made.

We thank Steve Wharton, MRC, Mill Hill, London, UK for assistance in the erythrocyte-binding assay and H.-D. Klenk and W. Garten, both from the University of Marburg, Germany, for bovine furin and WSN neuraminidase plasmids as well antiserum against the HA of influenza virus $\mathrm{A} / \mathrm{FPV} /$ Rostock/34 (H7N1).

\section{References}

Arthur, L. O., Bess, J. W., Jr, Sowder, R. C., II, Benveniste, R. E., Mann, D. L., Chermann, J. C. \& Henderson, L. E. (1992). Cellular proteins bound to immunodeficiency viruses: implications for pathogenesis and vaccines. Science 258, 1935-1938.

Bastiani, L., Laal, S., Kim, M. \& Zolla-Pazner, S. (1997). Host celldependent alterations in envelope components of human immunodeficiency virus type 1 virions. Journal of Virology 71, 3444-3450.

Blasco, R. \& Moss, B. (1995). Selection of recombinant vaccinia viruses on the basis of plaque formation. Gene 158, 157-162.

Bullough, P. A., Hughson, F. M., Skehel, J. J. \& Wiley, D. C. (1994). Structure of influenza haemagglutinin at the $\mathrm{pH}$ of membrane fusion. Nature 371, 37-43.

Daniels, R. S., Douglas, A. R., Skehel, J. J. \& Wiley, D. C. (1983). Analyses of the antigenicity of influenza haemagglutinin at the $\mathrm{pH}$ optimum for virus-mediated membrane fusion. Journal of General Virology 64, 1657-1662.

Dong, J., Roth, M. G. \& Hunter, E. (1992). A chimeric avian retrovirus containing the influenza virus hemagglutinin gene has an expanded host range. Journal of Virology 66, 7374-7382.

Ellens, H., Bentz, J., Mason, D., Zhang, F. \& White, J. M. (1990). Fusion of influenza hemagglutinin-expressing fibroblasts with glycophorinbearing liposomes: role of hemagglutinin surface density. Biochemistry 29, 9697-9707.

Geijtenbeek, T. B., Kwon, D. S., Torensma, R., van Vliet, S. J., van Duijnhoven, G. C., Middel, J., Cornelissen, I. L., Nottet, H. S., KewalRamani, V. N., Littman, D. R., Figdor, C. G. \& van Kooyk, Y. (2000). DC-SIGN, a dendritic cell-specific HIV-1-binding protein that enhances trans-infection of T cells. Cell 100, 587-597.

Hammarstedt, M., Wallengren, K., Pedersen, K. W., Roos, N. \& Garoff, H. (2000). Minimal exclusion of plasma membrane proteins during retroviral envelope formation. Proceedings of the National Academy of Sciences, USA 97, 7527-7532.

Hatziioannou, T., Valsesia-Wittmann, S., Russell, S. J. \& Cosset, F. L. (1998). Incorporation of fowl plague virus hemagglutinin into murine leukemia virus particles and analysis of the infectivity of the pseudotyped retroviruses. Journal of Virology 72, 5313-5317.

Henriksson, P. \& Bosch, V. (1998). Inhibition of cellular glycoprotein incorporation into human immunodeficiency virus-like particles by coexpression of additional cellular interaction partner. Virology 251, 16-21.

Henriksson, P., Pfeiffer, T., Zentgraf, H., Alke, A. \& Bosch, V. (1999). Incorporation of wild-type and $\mathrm{C}$-terminally truncated human epidermal 
growth factor receptor into human immunodeficiency virus-like particles: insight into the processes governing glycoprotein incorporation into retroviral particles. Journal of Virology 73, 9294-9302.

Ho, S. N., Hunt, H. D., Horton, R. M., Pullen, J. K. \& Pease, L. R. (1989). Site-directed mutagenesis by overlap extension using the polymerase chain reaction. Gene 77, 51-59.

Horton, R. M., Hunt, H. D., Ho, S. N., Pullen, J. K. \& Pease, L. R. (1989). Engineering hybrid genes without the use of restriction enzymes: gene splicing by overlap extension. Gene 77, 61-68.

Lama, J., Mangasarian, A. \& Trono, D. (1999). Cell-surface expression of CD4 reduces HIV-1 infectivity by blocking Env incorporation in a Nefand Vpu-inhibitable manner. Current Biology 9, 622-631.

Lin, Y. P., Wharton, S. A., Martin, J., Skehel, J. J., Wiley, D. C. \& Steinhauer, D. A. (1997). Adaptation of egg-grown and transfectant influenza viruses for growth in mammalian cells: selection of hemagglutinin mutants with elevated $\mathrm{pH}$ of membrane fusion. Virology 233, 402-410.

Liu, C., Eichelberger, M. C., Compans, R. W. \& Air, G. M. (1995). Influenza type A virus neuraminidase does not play a role in viral entry, replication, assembly, or budding. Journal of Virology 69, 1099-1106.

Martin, J., Wharton, S. A., Lin, Y. P., Takemoto, D. K., Skehel, J. J., Wiley, D. C. \& Steinhauer, D. A. (1998). Studies of the binding properties of influenza hemagglutinin receptor-site mutants. Virology 241, 101-111.

Melkonian, K. A., Ostermeyer, A. G., Chen, J. Z., Roth, M. G. \& Brown, D. A. (1999). Role of lipid modifications in targeting proteins to detergent-resistant membrane rafts. Many raft proteins are acylated, while few are prenylated. Journal of Biological Chemistry 274, 3910-3917.

Nguyen, D. H. \& Hildreth, J. E. (2000). Evidence for budding of human immunodeficiency virus type I selectively from glycolipid-enriched membrane lipid rafts. Journal of Virology 74, 3264-3272.

Ohuchi, R., Ohuchi, M., Garten, W. \& Klenk, H.-D. (1991). Human influenza virus hemagglutinin with high sensitivity to proteolytic activation. Journal of Virology 65, 3530-3537.

Ohuchi, M., Feldmann, A., Ohuchi, R. \& Klenk, H.-D. (1995). Neuraminidase is essential for fowl plague virus hemagglutinin to show hemagglutinating activity. Virology 212, 77-83.

Ohuchi, M., Ohuchi, R., Feldmann, A. \& Klenk, H.-D. (1997). Regulation of receptor binding affinity of influenza virus hemagglutinin by its carbohydrate moiety. Journal of Virology 71, 8377-8384.
Pear, W. S., Nolan, G. P., Scott, M. L. \& Baltimore, D. (1993). Production of high-titer helper-free retroviruses by transient transfection. Proceedings of the National Academy of Sciences, USA 90, 8392-8396.

Pfeiffer, T., Zentgraf, H., Freyaldenhoven, B. \& Bosch, V. (1997). Transfer of endoplasmic reticulum and Golgi retention signals to human immunodeficiency virus type 1 gpI60 inhibits intracellular transport and proteolytic processing of viral glycoprotein but does not influence the cellular site of virus particle budding. Journal of General Virology 78, 1745-1753.

Rittner, K., Stöppler, H., Pawlita, M. \& Sczakiel, G. (1991). Versatile eucaryotic vectors for strong and constitutive transient and stable gene expression. Methods in Molecular and Cellular Biology 2, 176-181.

Scheiffele, P., Roth, M. G. \& Simons, K. (1997). Interaction of influenza virus haemagglutinin with sphingolipid-cholesterol membrane domains via its transmembrane domain. EMBO Journal 16, 5501-5508.

Schnell, M. J., Johnson, J. E., Buonocore, L. \& Rose, J. K. (1997). Construction of a novel virus that targets HIV-1-infected cells and controls HIV-I infection. Cell 90, 849-857.

Schoen, P., Leserman, L. \& Wilschut, J. (1996). Fusion of reconstituted influenza virus envelopes with liposomes mediated by streptavidin/ biotin interactions. FEBS Letters 390, 315-318.

Steinhauer, D. A., Wharton, S. A., Wiley, D. C. \& Skehel, J. J. (1991). Deacylation of the hemagglutinin of influenza A/Aichi/2/68 has no effect on membrane fusion properties. Virology 184, 445-448.

Vey, M., Schafer, W., Berghofer, S., Klenk, H.-D. \& Garten, W. (1994). Maturation of the trans-Golgi network protease furin: compartmentalization of propeptide removal, substrate cleavage, and COOH-terminal truncation. Journal of Cell Biology 127, 1829-1842.

Wharton, S. A., Skehel, J. J. \& Wiley, D. C. (1986). Studies of influenza haemagglutinin-mediated membrane fusion. Virology 149, 27-35.

Wilson, I. A., Skehel, J. J. \& Wiley, D. C. (1981). Structure of the haemagglutinin membrane glycoprotein of influenza virus at $3 \AA$ resolution. Nature 289, 366-373.

Young, J. A., Bates, P., Willert, K. \& Varmus, H. E. (1990). Efficient incorporation of human $\mathrm{CD} 4$ protein into avian leukosis virus particles. Science 250, 1421-1423.

Zhang, J., Pekosz, A. \& Lamb, R. A. (2000). Influenza virus assembly and lipid raft microdomains: a role for the cytoplasmic tails of the spike glycoproteins. Journal of Virology 74, 4634-4644.

Received 8 March 2001; Accepted 25 June 2001 